\title{
Magnetic Field in the Proto-Planetary Nebula OH17.7-2.0
}

\author{
Indra Bains, Tim Gledhill \\ Astronomy Group, Department of Physical Sciences, University of \\ Hertfordshire, College Lane, Hatfield, Hertfordshire, AL10 9AB, UK \\ Jeremy Yates \\ Department of Physics and Astronomy, University College London, \\ Gower Street, London, WC1E 6BT, UK \\ Anita Richards \\ Jodrell Bank Observatory, Department of Physics $\&$ Astronomy, \\ University of Manchester, Macclesfield, Cheshire SK11 9DL, UK
}

\begin{abstract}
We have used radio observations of $\mathrm{OH}$ masers in protoplanetary nebula (PPN) candidates to probe the magnetic field structure of these objects. Here we present the first results of our study, concerning the PPN OH17.7-2.0.
\end{abstract}

\section{Introduction}

The collimation mechanism that is responsible for the production of bipolar planetary nebulae $(\mathrm{PN})$ is yet to be understood. Current theories regarding these mechanisms detail the potential rôle played by magnetic fields. In evolved stars, the $1612 \mathrm{MHz}$ and $1667 \mathrm{MHz}$ masing $\mathrm{OH}$ species have been shown to trace outflow regions (e.g. Sahai et al. 1999) and the remnant AGB shell (e.g. Seaquist \& Davis 1983). These species are also tracers of magnetic fields, their Zeeman split emission lines giving information on the structure, magnitude and direction of the fields (e.g. Chapman \& Cohen 1986; Szymczak, Cohen, \& Richards 2001).

We have obtained full polarization MERLIN spectral line observations of a sample of PPN at $1612 \mathrm{MHz}$ and $1667 \mathrm{MHz}$ with a view to probing their magnetic field structure. In this poster we present our first results, concerning observations of $\mathrm{OH}$ masers in $\mathrm{OH} 17.7-2.0$.

\section{Results}

At both observing frequencies (beamsize $0^{\prime \prime} .3 \times 0^{\prime \prime} .2$ ), the Stokes I maser components are distributed in a general NW-SE direction. The spatial distribution of the linearly polarized maser components detected at each frequency is shown in Figure 1. The linear polarization vectors are highly ordered; at $1612 \mathrm{MHz}$ they are all within $\sim 30^{\circ}$ of E-W alignment, indicating an ordered magnetic field structure. 

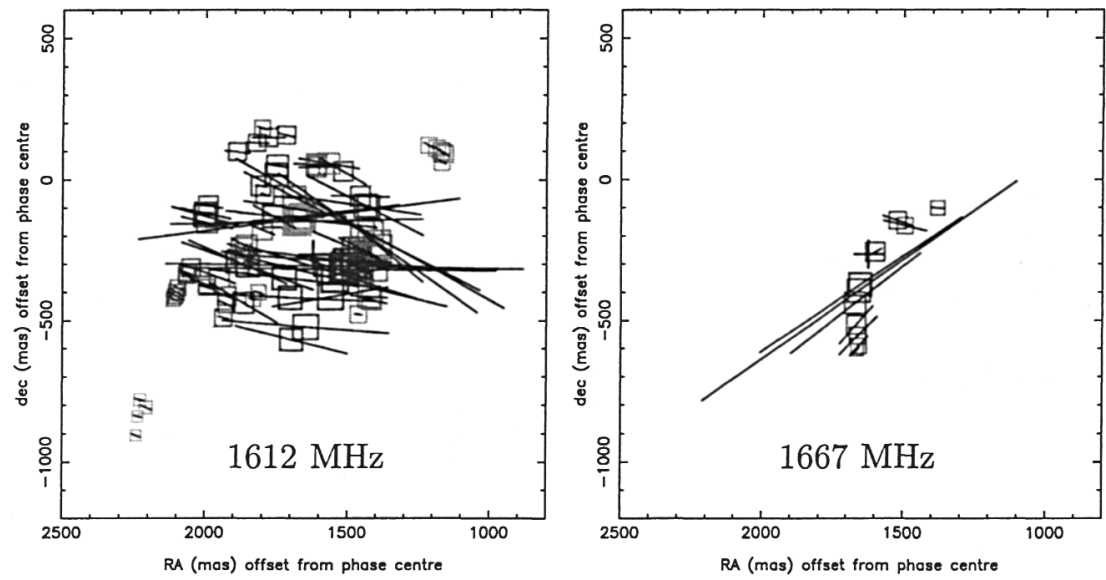

Figure 1. Spatial distribution of linearly polarized maser components. Symbol size is proportional to the logarithm of the peak total flux density, vector magnitude $\left(1^{\prime \prime} \approx 0.9 \mathrm{Jy} \mathrm{beam}^{-1}\right)$ is proportional to the peak linearly polarized flux density. Lighter spots are more blueshifted and darker more redshifted. The estimated centre of expansion of the masers is indicated by a cross.

One candidate for a blended Zeeman split feature was found at each observing frequency. From these we estimate a magnetic field strength of $\leq+4.6 \mathrm{mG}$ $(1612 \mathrm{MHz})$ and $\leq+2.5 \mathrm{mG}(1667 \mathrm{MHz})$. The positive sign indicates that the field is directed away from the Earth.

Further details can be found in the forthcoming paper, Bains et al., in prep.

Acknowledgments. We wish to thank the MERLIN staff for their advice regarding the data reduction. I. B. acknowledges a PPARC PDRA grant.

\section{References}

Chapman, J. M., \& Cohen, R. J. 1986, MNRAS, 220, 513

Sahai, R., Te Lintel Hekkert, P., Morris, M., Zijlstra, A., \& Likkel, L. 1999, ApJ, 514, L115

Seaquist, E. R., \& Davis L. E. 1983, ApJ, 274, 659

Szymczak, M., Cohen, R. J., Richards, A. M. S. 2001, A\&A, 371, 1012 